\title{
Variable Data Rate for Free Space Optical Low Earth Orbit Downlinks (OLEODL)
}

\author{
Amita Shrestha, Dirk Giggenbach, and Norbert Hanik* \\ Deutsches Zentrum für Luft und Raumfahrt e.V., Münchner Straße 20, 82234 Weßling, Germany \\ * Technische Universität München, Theresienstrasse 90, 80290 München, Germany \\ Tel: 08153 28-3574, Fax:08153 28-2844, e-mail: amita.shrestha@dlr.de
}

\begin{abstract}
Low-Earth-orbit (LEO) satellites for Earth observation have typical orbit altitudes of 400 to $600 \mathrm{~km}$. Since their on-board sensors resolution is advancing, the need for very high rate satellite downlinks is increasing into the range of several gigabits per second. Free-space optical communications is ideal for such applications, as it offers very high data rate, while allowing smaller satellite terminals and avoiding spectral regulation constraints. However, the key challenges in optical LEO downlinks (OLEODL) are the varying free space loss due to distance changes during a downlink contact, atmospheric attenuation, and the fast signal scintillation due to atmospheric index-of-refraction turbulence (IRT). This paper will briefly explain OLEODL technology, its challenges, and suitable mitigation techniques. The paper will focus on why varying the data rate according to channel state is important for OLEODL and how it can be achieved.
\end{abstract}

Keywords: variable data rate, OLEODL, optical communication, satellite communication, laser communication.

\section{INTRODUCTION AND BACKGROUND}

The demand for very high speed LEO downlink is increasing because of advanced development of high resolution on-board sensors, mainly used for earth observations. Free-space optical (FSO) communication is an ideal solution because of its high throughput possibility, less power and small terminal size requirement, high security, and no limitation in the bandwidth. However, FSO channel has challenging atmospheric effects that deteriorate the transmitted optical beam as shown in Fig. 1, thereby causing signal scintillation. In addition, the signal is also affected by fog, rain, background light due to sunlight, and transient misspointing. Some of the widely used techniques to minimize such challenges are aperture averaging, adaptive optics, spatial transmitter diversity, wavelength diversity, forward error correction together with a long interleaver, and variable data rate [1]. This paper explains the variable data rate and some options how it can be realised in OLEODL scenario.

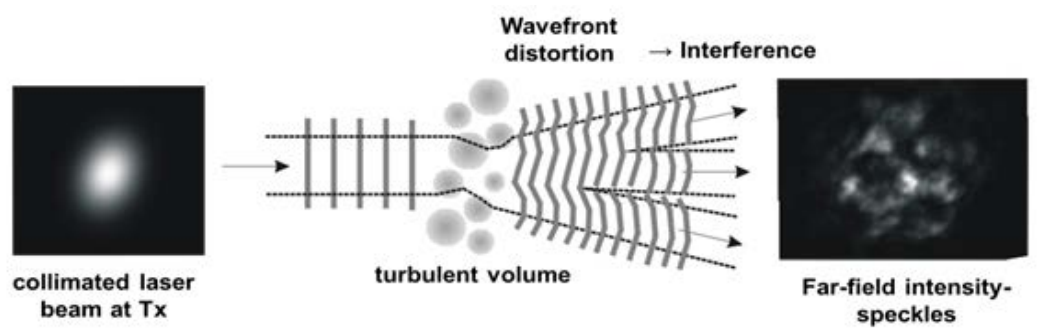

Figure 1. Laser beam transmission through free-space optical channel.

Link geometry of typical LEO satellites is shown in Fig. 2. The satellite orbits around the earth at a distance that is $400 \mathrm{~km}$ to $900 \mathrm{~km}$ above the Earth surface. An OLEODL session includes pointing, acquisition and tracking (PAT) phases. PAT phases can be explained with the help of Fig. 3 In the beginning, when the satellite appears above the horizon, both the satellite and the Ground Station (GS) point towards each other using the known position of the GS and satellite's orbit information. The GS illuminates the satellite by transmitting an uplink beacon. Then the satellite acquires this signal, corrects its attitude, and illuminates the GS with the communication signal. The GS then acquires this signal, corrects its pointing, and continues closed loop tracking. Finally, line of sight pointing and tracking between the communications partners is maintained and data transmission starts. The data can be transmitted as long as satellite and GS are in line of sight without clouds blocking the link and the link being at a certain minimum elevation above the horizon. In order to maximize the total contact time of the satellite, an optical downlink should start acquisition of the optical signals at around $5^{\circ}$ elevation, and secure data transmission should work from $10^{\circ}$ elevation upwards. This is also the typical operation range for a RF communication link. 


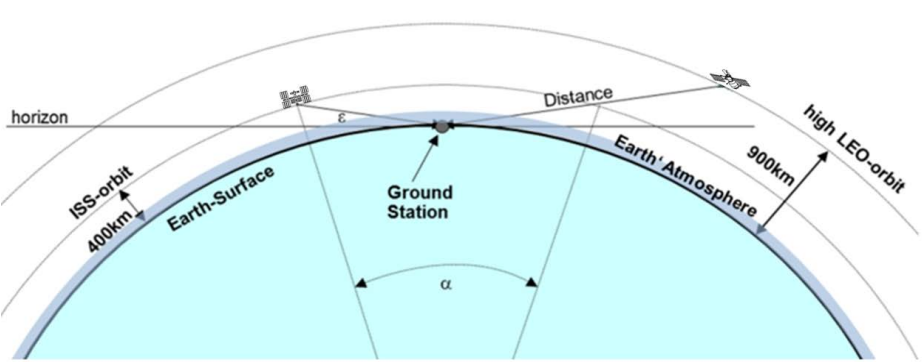

Figure 2. Link geometry of typical satellite downlinks with circular orbits. For e.g. ISS orbit which is $400 \mathrm{~km}$ and high LEOOrbit which is $900 \mathrm{~km}$ away from the earth's surface

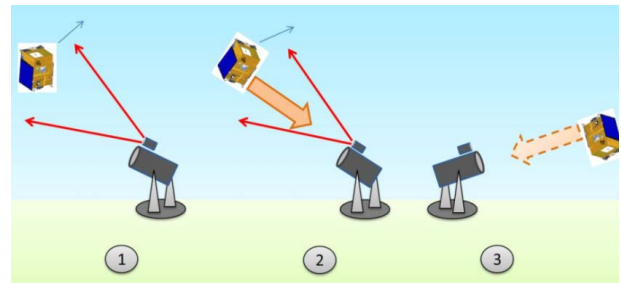

Figure 3. Typical OLEODL pass phases:

(1) Pointing and acquisition, (2) tracking and communications link, (3) link termination.

\section{OLEODL CHANNEL}

In the OLEODL scenario, the distance between the satellite and ground varies, therefore the link budget also varies. In addition, depending on the position of the satellite and link elevation angle, atmospheric attenuation and scintillation also varies [2]. At lower elevations, the signal passes through a longer atmospheric path, thus gets more attenuated and deteriorated. This was verified during experimental downlinks from the Japanese satellite OICETS to DLR's optical ground station in 2006 and 2009 [3]. Figure 4 depicts that at lower elevations, lower optical power is received, and higher scintillation index (measure of the signal fluctuation due to atmospheric effects), is observed. This implies that lower elevation angles are very critical compared to higher elevations. The situation can be improved by using larger telescopes, adaptive optics, very long and complex coding and interleaving techniques. However, applying such techniques constantly will result in waste of resources for the times when the channel condition is better i.e. at higher elevation. Study presented in paper [3] suggests that a system with capability of adaptive data-rate, code-rate, modulation scheme and interleaver size would be a more efficient approach to mitigate those atmospheric challenges. According to the study done in [4], with the implementation of an ideal adaptive data rate in a typical LEO orbit situation, throughput improvement of more than 3 times compared to optimum fixed data rate can be expected. However, the realistic factor could be slightly less as this calculation was done under several assumptions like the satellite having a low circular orbit, uniform position probability on its orbital sphere, no atmospheric turbulence, equivalent cloud probability, constant receiver sensitivity on photons per bit etc.
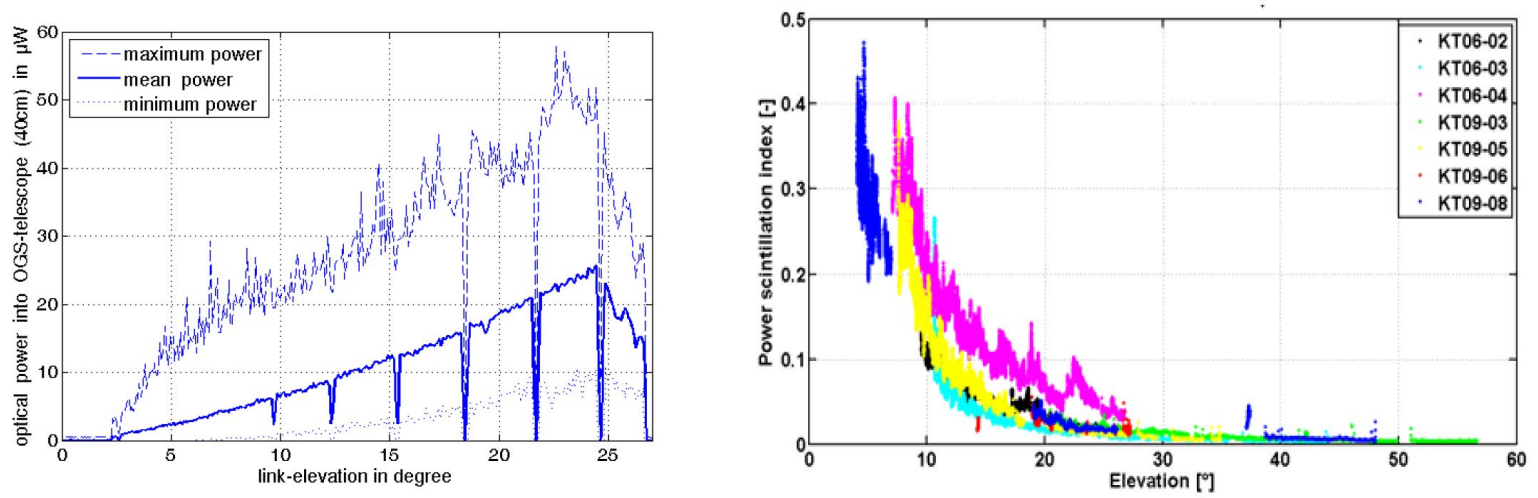

Figure 4. Optical power received at the detector (left) and power scintillation index (right) with respect to elevation measured during the KIODO downlinks [5]. In left figure, power starts to fall after $25^{\circ}$ elevation because of the cloud appearance, and periodical drops of the mean power is because of a tracking error.

\section{VARIABLE DATA RATE}

\subsection{Motivation for Variable Data Rate}

When the LEO satellite is in the line of sight of an OGS, its viewing elevation is restricted to low elevations most of the time. As stated in earlier sections, higher atmospheric disturbances are experienced at lower elevation angles. However, the study done in paper [5] shows that lower elevations are very important because they occupy significant part of the contact time. When defining $5^{\circ}$ as the minimum possible contact elevation, the satellite is seen between $5^{\circ}$ and $20^{\circ}$ for $45 \%$ of the total contact time as shown in Fig. 5. This has a major influence on the data format and link protection. For non-adaptive systems, very strong coding and interleaving techniques needs to be defined for error-free transmission which increases the redundancy for the time when 
channel condition is good at higher elevation angles. Therefore, operating the system at lower data rate at lower elevation and maximum possible data rate at zenith is a very efficient solution.

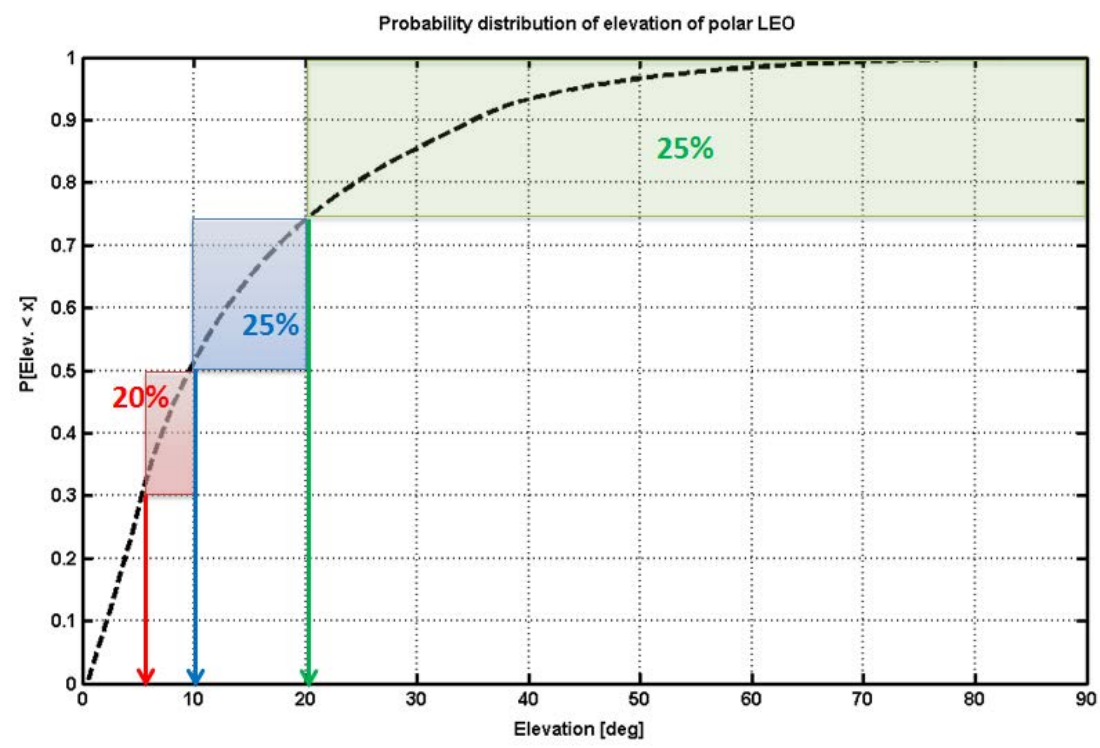

Figure 5. Typical distribution of the average viewing elevation for a polar LEO satellite (500 km orbit height). This relative distribution is qualitatively similar for any OGS-location on earth, although of course the absolute overall visibility changes depending on orbit and OGS latitude (Taken from Fig. 3 of [5]).

Moreover, different modes of varying the data rate in an OLEODL-system can be identified:

a) While a specific satellite terminal might only work at one data rate, still a GS may need to vary its receiver rate since it serves different types of satellite missions.

b) A constant rate during one downlink contact is chosen according to its pass geometry, e.g. to allow maximum throughput during this link.

c) Depending on the progression of the link elevation, the transmitter varies the effective data rate on preprogrammed time steps, to adopt to the known elevation-dependent link losses.

By exchanging channel state information (CSI) between ground and satellite, the optimum rate is chosen dynamically, every time the link budget changes notably.

\subsection{Variation of Data Rate for a Variable Link Budget}

Targeted data rates in OLEODL range from a few megabits per second for very simple and low-cost satellites and terminals with limited pointing control and transmit power, to several gigabits per second for high throughput Earth observation sensor data downloads. Assuming the low cost OLEODL scenario, maximum data rate of $10 \mathrm{Gbps}$ can be considered, as at this rate multi-mode photo detectors are available to date. An optimised data throughput depends on the variation of the rate due to link constraints such as channel attenuation and power variation as caused by the index-of-refraction turbulence (IRT) of the atmosphere. According to the study done in [5], assuming a receiver that performs with constant energy/bit, a link from $5^{\circ}$ elevation to zenith allows a rate variation of around 25 as shown in Fig. 6. This plot includes the elevation-dependent atmospheric signal attenuation, but not the dynamic scintillation and fading effects caused by atmospheric turbulence. Data rate variation can be achieved by varying only the source data rate and maintaining the symbol rate, while, some techniques involve varying the channel symbol rate as well. Different options to vary the data rates are listed in Fig. 7 and explained below.

\subsubsection{Varying the Channel Symbol Rate}

The variation of bits per channel symbol with On-Off-Keying (OOK) modulation can be done with a PulsePosition (PPM) or Amplitude-Shift-Keying (ASK) modulation format, where one pulse transmits the information of more than one bit. One of the simplest ways to vary the effective data rate is to alter the length of one symbol time. However, this requires the change in the receiver hardware to match the bandwidth of the receiver to the pulse length, which is not feasible to support the data rate variation within one link.

Another option would be to use RZ-OOK and alter the duty cycle but maintaining the pulse length. In addition, multi order PPM can be used by changing the PPM order according to the effective data rate and keeping the pulse length constant. Both options avoid the need for the change in receiver hardware but increase the complexity of the system. The performance analysis of these options are presented in [6] and [7], and is out of scope of this paper. 


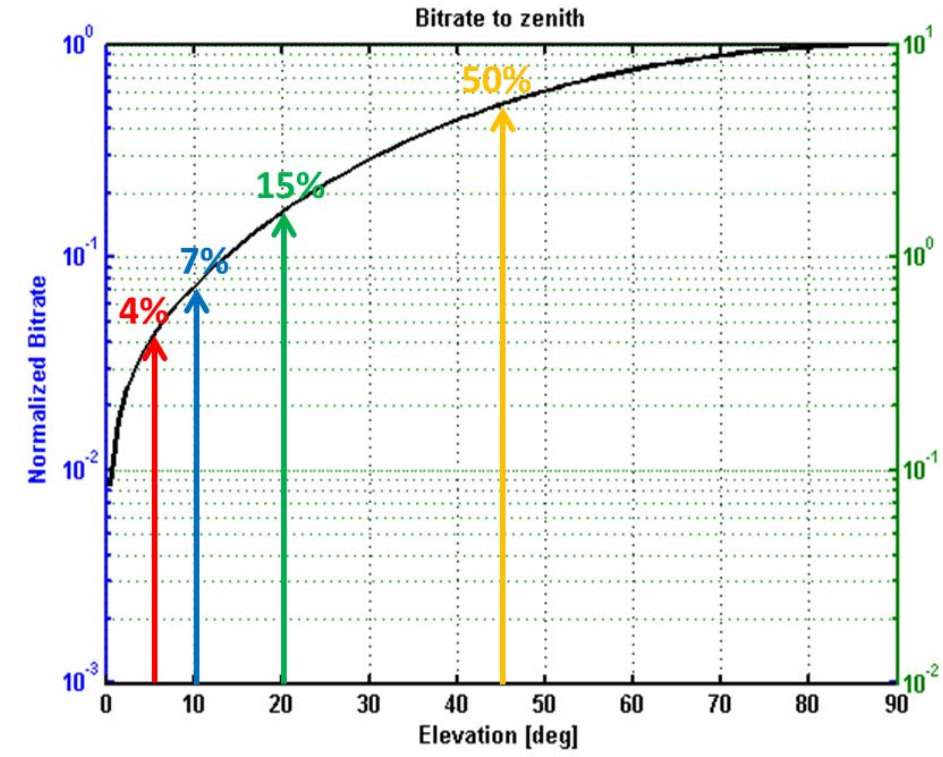

Figure 6. Downlink bitrate normalised to zenith, for constant energy per bit (i.e. sensitivity per bit is independent of data rate), including range- and atmospheric losses [5].

\subsubsection{Varying the Source Data Rate but Maintaining the Symbol Rate}

Variations of effective source data rates do not necessarily require a change in channel symbol rate and thus receiver hardware. FEC (Forward Error Correction) is the standard technique used to protect data against bit errors in a simplex link. The source data rate can be varied simply by varying the coding overhead. This allows the variation of data rate in addition to the variation in FEC-gain. Other options such as burst transmission with pauses between data sections, frame repetition, and interleaver techniques are partly advantageous in a fading channel. These mechanisms are used in different sophistication levels of rate variation modes, in order to maximise the overall downlink system throughput under varying link loss, while also securing a frequent access to the satellite. Similar gain can also be achieved by retransmitting the frames/codewords after certain delay that is greater than the fading length of the channel. The technique is named Delayed Frame Retransmission (DFR) and briefly explained in [7]. This technique is proven to provide even a net sensitivity gain when used for fading channel with higher scintillation index. Moreover, more effective approach would be to spread the codeword instead of repeating the whole codeword as in case of DFR, provided the delay between different parts of the codeword is longer than the fading length of the channel.

\section{CONCLUSIONS}

Free space optical LEO downlinks fulfil the increasing demand of very high data rate from Earth Observation LEO satellites. However, OLEODL has two key challenges: varying link budget due to distance and atmospheric attenuation, and fast power scintillation due to atmospheric IRT. Varying the data rate according to the channel is the ideal solution to cope with these challenges, and it also increases the overall throughput of the link by allowing data transmission even at lower elevations. Data rate variation can be achieved by using different options that either vary the symbol rate and the source data rate or only the source data rate, keeping the channel symbol rate constant.

\section{REFERENCES}

[1] M. A. Khalighi and M. Uysal, "Survey on free space optical communication: A communication theory perspective," IEEE Communications Surveys and Tutorials, 2013.

[2] D. Giggenbach and F. Moll, "Scintillation Loss in optical low earth orbit data downlinks with avalanche photodiode receivers," in International Conference on Space Optical Systems (ICSOS), 2017.

[3] F. Moll and M. Knapek, "Free-space laser communications for satellite downlinks: Measurements of the atmospheric channel," in Proc. International Aeronautical Congress (IAC), 2011.

[4] N. Perlot and T. de Cola, "Throughput Maximization of optical LEO-ground links," in Proc. SPIE, FreeSpace Laser Communication Technologies XXIV, 2012, vol. 8246.

[5] D. Giggenbach, F. Moll, C. Fuchs, T. de Cola, and R. M. Calvo, "Space communications protocols for future optical satellite downlinks," in Proc. International Astonautical Congress (IAC), 2011.

[6] A. Shrestha and D. Giggenbach, "Variable data rate for optical low-earth-orbit (LEO) downlinks," in ITGFachbericht 264: Photonische Netze, 2016.

[7] A. Shrestha and D. Giggenbach, "Delayed frame repetition for free space optical communication (FSO) channel," in ITG-Fachbericht, vol. 272, pp. 44-50, 2017. 